JOURNAL OF THE

AMERICAN MATHEMATICAL SOCIETY

Volume 11, Number 1, January 1998, Pages 175-188

S 0894-0347(98)00251-3

\title{
ASYMPTOTIC PROPERTIES OF BANACH SPACES UNDER RENORMINGS
}

\author{
E. ODELL AND TH. SCHLUMPRECHT
}

\section{§1. IntRoduCtion}

A classical problem in functional analysis has been to give a geometric characterization of reflexivity for a Banach space. The first result of this type was D.P. Milman's [Mil] and B.J. Pettis' [P] theorem that a uniformly convex space is reflexive. While perhaps considered elementary today it illustrated how a geometric property can be responsible for a topological property. Of course a Banach space can be reflexive without being uniformly convex, even under renormings, as shown by M.M. Day [D2]. The problem considered for years by functional analysts was whether there exists a weaker property of a geometric nature which is equivalent to reflexivity. In this paper we give an affirmative solution by demonstrating that such a property exists. The property was suggested in 1971 by V.D. Milman [Mi] (see also [DGZ], problem IV, p.177). We prove that a separable Banach space $X$ is reflexive (if and) only if there exists an equivalent norm $\|\cdot\|$ on $X$ so that

$$
\begin{aligned}
& \text { whenever a sequence }\left(x_{n}\right) \subseteq X \text { satisfies } \\
& \lim _{n} \lim _{m}\left\|x_{n}+x_{m}\right\|=2 \lim _{n}\left\|x_{n}\right\| \\
& \text { then }\left(x_{n}\right) \text { must converge in norm. }
\end{aligned}
$$

The "if" part of the characterization follows easily from James' famous characterization of reflexivity in terms of the sup of linear functionals [J1]. Indeed given $x^{*} \in X^{*}$ with $\left\|x^{*}\right\|=1$ choose $\left(x_{n}\right) \subseteq X$ with $x^{*}\left(x_{n}\right) \rightarrow 1$ and $\left\|x_{n}\right\|=1$ for all $n$. Then $\lim _{m} \lim _{n}\left\|x_{n}+x_{m}\right\|=2$ and so $x_{n} \rightarrow x$ with $\|x\|=1$. Thus $x^{*}(x)=1$ so $x^{*}$ attains its norm. Hence by [J1] $X$ is reflexive.

The investigation of spaces having property $(*)$ (also called property $(2 R)$ in [D1]) goes back to the 1950's. In [FG], for example, the relation of $(*)$ to other smoothness and rotundity properties was studied. For a more complete survey of these notions we refer the reader to [DGZ].

More recently (over the past 30 years) functional analysts have considered the question as to what sort of nice infinite dimensional subspaces one can find in an arbitrary infinite dimensional Banach space $X$. One can assume $X$ has a basis and ask: What kinds of block bases does it have? Must one be unconditional? Is some block subspace either reflexive or isomorphic to $c_{0}$ or $\ell_{1}$ ? These problems are related. James [J3] showed that if $\left(x_{i}\right)$ is an unconditional basis for $X$, then

Received by the editors May 12, 1997 and, in revised form, September 15, 1997.

1991 Mathematics Subject Classification. Primary 46B03, 46B45.

Key words and phrases. Spreading model, Ramsey theory, $\ell_{1}, c_{0}$, reflexive Banach space. Research of both authors was supported by NSF and TARP.

(C) 1998 American Mathematical Society 
either $X$ is reflexive or some block basis is equivalent to the unit vector basis for $c_{0}$ or $\ell_{1}$. W.T. Gowers [G1] proved the following remarkable dichotomy theorem: $X$ contains a subspace $Y$ which either has an unconditional basis or is H.I. (hereditarily indecomposable; i.e., if $Z \subseteq Y$ and $Z=V \oplus W$, then $V$ or $W$ must be finite dimensional). Gowers and Maurey $[\mathrm{GM}]$ proved that both alternatives are possible. Then Gowers [G2] proved that a space need not contain $c_{0}, \ell_{1}$ or a reflexive subspace.

The search for an answer to this last problem led to much research into both characterizations of reflexivity and to the characterization as to when $X$ contains isomorphs of $c_{0}$ or $\ell_{1}$ (e.g., [J1], [J2], [J3], [R1], [R2], [R3], [BP], [M]). The proof of our characterization of reflexivity led to additional characterizations as to when $X$ contains $c_{0}$ or $\ell_{1}$ in terms of the asymptotic behavior of sequences in $X$.

There are two main notions of asymptotic properties in Banach spaces. The first is that of a spreading model. If $\left(x_{n}\right)$ is bounded in $X$, then by using Ramsey theory (cf. $[\mathrm{B}],[\mathrm{BS}],[\mathrm{O}],[\mathrm{BL}]$ ) one can extract a subsequence $\left(y_{n}\right)$ so that for all $k$ and $\left(a_{i}\right)_{1}^{k} \subseteq \mathbb{R}$, we have the existence of the iterated limit

$$
\lim _{n_{1} \rightarrow \infty} \ldots \lim _{n_{k} \rightarrow \infty}\left\|\sum_{i=1}^{k} a_{i} y_{n_{i}}\right\| \equiv f\left(a_{1}, \ldots, a_{k}\right) .
$$

If $\left(y_{n}\right)$ does not converge in the norm topology, then $f(\cdot)$ is a norm on $c_{00}$, the linear space of all finitely supported real valued sequences. Let $\left(e_{i}\right)$ be the unit vector basis of $c_{00}$. If $\left(y_{n}\right)$ does not converge weakly to a nonzero element of $X$, then $\left(e_{i}\right)$ is a basis for $E=\left[\left(e_{i}\right)\right]$, the completion of $c_{00}$ under $f(\cdot)$. In this case we call $\left(e_{i}\right)$ or $E$ the spreading model of $\left(y_{n}\right)$. If $\left(x_{i}\right)$ is weakly null, then the spreading model $\left(e_{i}\right)$ is unconditional. In any event the spreading model is subsymmetric $\left(\left\|\sum a_{i} e_{i}\right\|=\left\|\sum a_{i} e_{n_{i}}\right\|\right.$ if $\left(a_{i}\right) \subseteq \mathbb{R}$ and $\left.n_{1}<n_{2}<\cdots\right)$ and $\left(e_{1}-e_{2}, e_{3}-e_{4}, \ldots\right)$ is unconditional.

The second notion of asymptotic structure is due to Maurey, Milman and Tomczak-Jaegermann (see $[\mathrm{MT}],[\mathrm{MMT}]$ ). Let $X$ have a basis $\left(x_{i}\right)$. For $x, y \in X$ we write $x<y$ if $\max \operatorname{supp} x<\min \operatorname{supp} y$ where if $x=\sum a_{i} x_{i}$, then $\operatorname{supp} x=$ $\left\{i: a_{i} \neq 0\right\} .\left\langle x_{i}\right\rangle_{i \in I}$ denotes the linear span of $\left\{x_{i}: i \in I\right\}$ and $S_{\left\langle x_{i}\right\rangle_{i \in I}}$ denotes the unit sphere of this span. Let $n \in \mathbb{N}$ and let $\left(w_{i}\right)_{1}^{n}$ be a normalized basis for some $n$ dimensional space. We say $\left(w_{i}\right)_{1}^{n} \in\{X\}_{n}$ if

$$
\begin{aligned}
& \forall k_{1} \in \mathbb{N} \exists y_{1} \in S_{\left\langle x_{i}\right\rangle_{k_{1}}^{\infty}} \forall k_{2} \in \mathbb{N} \\
& \exists y_{2} \in S_{\left\langle x_{i}\right\rangle_{k_{2}}^{\infty}} \cdots \forall k_{n} \in \mathbb{N} \exists y_{n} \in S_{\left\langle x_{i}\right\rangle_{k_{n}}^{\infty}}
\end{aligned}
$$

so that $\left(y_{i}\right)_{1}^{n}$ is $1+\varepsilon$-equivalent to $\left(w_{i}\right)_{1}^{n}$. This means that there exist $A, B$ with $A B \leq 1+\varepsilon$ so that for all $\left(a_{i}\right)_{1}^{n} \subset \mathbb{R}$

$$
A^{-1}\left\|\sum_{1}^{n} a_{i} y_{i}\right\| \leq\left\|\sum_{1}^{n} a_{i} w_{i}\right\| \leq B\left\|\sum_{1}^{n} a_{i} y_{i}\right\| .
$$

Note that if $\left(e_{i}\right)$ is a spreading model of a normalized block basis of $\left(x_{i}\right)$, then $\left(e_{i}\right)_{1}^{n} \in\{X\}_{n}$ for all $n$.

Both notions give a more regular structure in general than that possessed by the original space $X$. They are a joining of the finite and infinite dimensional structures of the space. Generally only finite dimensional information can be gleaned about $X$ from knowledge of its asymptotic structure. 
For example, note that the Schreier space $S$ ([CS], p.1) has a basis having a spreading model isometric to $\ell_{1}$ and yet $S$ is $c_{0}$ saturated (all infinite dimensional subspaces of $S$ contain $c_{0}$ ). Tsirelson's space $T$ (the dual of Tsirelson's original space $[\mathrm{T}]$ as described in [FJ]; see also [CS]) has a basis with the property that all spreading models are isomorphic to $\ell_{1}$ and in addition every infinite dimensional subspace contains a sequence whose spreading model is isometric to $\ell_{1}[\mathrm{OS}]$. Yet $T$ is reflexive. We do have the following result which requires a very strong assumption on the class of spreading models.

Theorem ([OS]). If $\left(x_{i}\right)$ is a basis for $X$ and if every spreading model $\left(e_{i}\right)$ of any normalized block basis of $\left(x_{i}\right)$ is 1-equivalent to the unit vector basis of $\ell_{1}$ (respectively, $\left.c_{0}\right)$, then $X$ contains an isomorph of $\ell_{1}$ (respectively, $\left.c_{0}\right)$.

In this paper we deduce information about the infinite dimensional structure of $X$ from knowledge about its asymptotic structure under equivalent norms.

We shall show that a separable space $(X,\|\cdot\|)$ can be given a special renorming \|\|$\cdot \|$ so that certain information about a given spreading model $E$ yields information about the infinite dimensional structure of $X$. For example if $\left\|e_{i} \pm e_{2}\right\|=2$ (respectively, $\left\|e_{1}+e_{2}\right\|=1$ ) for some spreading model $\left(e_{i}\right)$ of a normalized (and respectively, weakly null) sequence in $X$, then $X$ contains $\ell_{1}$ (respectively, $c_{0}$ ). Furthermore we show that a subspace $Y$ of $X$ is reflexive iff $Y$ satisfies (*).

Our main result is the following theorem.

Main Theorem. Every separable Banach space $X$ admits an equivalent strictly convex norm $\|\cdot\|$ with the following properties.

a) If $\left(x_{m}\right) \subseteq X$ is relatively weakly compact and if

$$
\lim _{m \rightarrow \infty} \lim _{n \rightarrow \infty}\left\|x_{m}+x_{n}\right\|=2 \lim _{n \rightarrow \infty}\left\|x_{n}\right\|,
$$

then $\left(x_{n}\right)$ is norm convergent.

b) If $\left(x_{n}\right) \subseteq X$ satisfies

$$
\lim _{m \rightarrow \infty} \lim _{n \rightarrow \infty}\left\|x_{m} \pm x_{n}\right\|=2 \lim _{n \rightarrow \infty}\left\|x_{n}\right\|>0,
$$

then some subsequence of $\left(x_{n}\right)$ is equivalent to the unit vector basis of $\ell_{1}$.

c) If $\left(x_{n}\right) \subseteq X$ is weakly null and satisfies

$$
\lim _{m \rightarrow \infty} \lim _{n \rightarrow \infty}\left\|x_{m}+x_{n}\right\|=\lim _{n \rightarrow \infty}\left\|x_{n}\right\|>0,
$$

then some subsequence of $\left(x_{n}\right)$ is equivalent to the unit vector basis of $c_{0}$.

This theorem is proved in $\S 2$.

As a corollary we deduce Milman's suggested characterization of reflexivity. In addition we obtain that $X$ contains $\ell_{1}$ if under all equivalent norms, $Y$ admits a normalized basic sequence having a spreading model $\left(e_{i}\right)$ satisfying $\left\|e_{1} \pm e_{2}\right\|=2$. In particular if under all equivalent norms $X$ admits a spreading model $\left(e_{i}\right)$ which is 1-equivalent to the unit vector basis of $\ell_{1}$, then $X$ contains an isomorph of $\ell_{1}$. If under all equivalent norms $X$ admits a weakly null sequence having spreading model $\left(e_{i}\right)$ with $\left\|e_{1}+e_{2}\right\|=1$ (e.g., if $\left(e_{i}\right)$ is 1-equivalent to the unit vector basis of $c_{0}$ ), then $X$ contains an isomorph of $c_{0}$. From James' proof that $\ell_{1}$ and $c_{0}$ are not distortable [J2] one obtains that both implications can be reversed.

In $\S 3$ we present some corollaries discussed briefly in this introduction. Our notation is standard as may be found in [LT]. 


\section{$\S 2$. Proof of the Main Theorem}

We first recall the following results of Maurey and Rosenthal.

Theorem ([M], [R1]). Let $X$ be a separable Banach space.

a) $X$ is not reflexive if and only if there exists a normalized basic sequence $\left(x_{n}\right) \subseteq$ $X$ satisfying for all $x \in X$ and $\alpha, \beta \geq 0$ with $\alpha+\beta=1$,

$$
\lim _{m \rightarrow \infty} \lim _{n \rightarrow \infty}\left\|x+\alpha x_{n}+\beta x_{n}\right\|=\lim _{m \rightarrow \infty}\left\|x+x_{m}\right\| .
$$

b) $X$ contains an isomorph of $\ell_{1}$ if and only if there exists a normalized basic sequence $\left(x_{n}\right) \subseteq X$ such that for all $x \in X$ and $\alpha, \beta \in \mathbb{R}$ with $|\alpha|+|\beta|=1$,

$$
\lim _{m \rightarrow \infty} \lim _{n \rightarrow \infty}\left\|x+\alpha x_{m}+\beta x_{n}\right\|=\lim _{m \rightarrow \infty}\left\|x+x_{m}\right\| \text {. }
$$

c) $X$ contains an isomorph of $c_{0}$ iff there exists a normalized basic sequence $\left(x_{n}\right) \subseteq X$ such that for all $x \in X$ and $\alpha, \beta \in \mathbb{R}$ with $|\alpha| \vee|\beta|=1$,

$$
\lim _{m \rightarrow \infty} \lim _{n \rightarrow \infty}\left\|x+\alpha x_{m}+\beta x_{n}\right\|=\lim _{m \rightarrow \infty}\left\|x+x_{m}\right\| \text {. }
$$

The intuition behind these results and the techniques employed to prove them had their origin in $[\mathrm{KM}]$ where types were introduced (and further developed in [HM]). A type $\tau_{\left(x_{n}\right)}$ on $X$ is a function on $X$ defined by a bounded sequence $\left(x_{n}\right) \subseteq X, \tau_{\left(x_{n}\right)}(x)=\lim _{n \rightarrow \infty}\left\|x+x_{n}\right\|$. Types give information on the asymptotic behavior of a sequence acting on the whole space. This contrasts with the notion of a spreading model which involves only the asymptotic behavior of the sequence $\left(x_{n}\right)$ itself. In this paper we characterize the three properties considered in the theorem above in terms solely of the asymptotic behavior of the sequences themselves. The price that must necessarily be paid is that we have to consider this behavior under all equivalent norms on $X$.

Let $(X,\|\cdot\|)$ be a Banach space over $\mathbb{R}$. If $x \in X$ we define the symmetrized type norm $\|\cdot\|_{x}: X \rightarrow[0, \infty)$ by

$$
\|y\|_{x}=\|x\| y\|+y\|+\|x\| y\|-y\| \text { for } y \in X .
$$

Lemma 2.1. For all $x \in X,\|\cdot\|_{x}$ is an equivalent norm on $X$ satisfying $2\|y\| \leq$ $\|y\|_{x} \leq 2(1+\|x\|)\|y\|$ for all $y \in X$.

Proof. The only property not evident is that $\|\cdot\|_{x}$ satisfies the triangle inequality. It is easy to check that for fixed $u, v \in X$ the function $r \mapsto\|r u+v\|+\|r u-v\|$ is symmetric and convex on $\mathbb{R}$ and thus increasing on $[0, \infty)$. Hence for $y_{1}, y_{2} \in X$

$$
\begin{aligned}
\left\|y_{1}+y_{2}\right\|_{x} & =\|x\| y_{1}+y_{2}\left\|+y_{1}+y_{2}\right\|+\|x\| y_{1}+y_{2}\left\|-y_{1}-y_{2}\right\| \\
& \leq\left\|x\left(\left\|y_{1}\right\|+\left\|y_{2}\right\|\right)+y_{1}+y_{2}\right\|+\left\|x\left(\left\|y_{1}\right\|+\left\|y_{2}\right\|\right)-y_{1}-y_{2}\right\| \\
& \leq\|x\| y_{1}\left\|+y_{1}\right\|+\|x\| y_{2}\left\|+y_{2}\right\|+\|x\| y_{1}\left\|-y_{1}\right\|+\|x\| y_{2}\left\|-y_{2}\right\| \\
& =\left\|y_{1}\right\|_{x}+\left\|y_{2}\right\|_{x} .
\end{aligned}
$$

Let $X$ be a separable Banach space. It is well known that $X$ admits an equivalent strictly convex norm $\|\cdot\|$, i.e., $\|x\|=\|y\|=1$ and $\|x+y\|=2$ implies that $x=y$. Fix a countable dense subset $C$ in $X$ which is closed under rational linear combinations. Choose $\left(p_{c}\right)_{c \in C} \subseteq(0, \infty)$ so that $\sum_{c \in C} p_{c}(1+\|c\|)<\infty$ for some (and thus for any) equivalent norm on $X$. If $\|\cdot\|$ is an equivalent norm on $X$, define $\|\cdot\|: X \rightarrow[0, \infty)$ 
by $\|x\|=\sum_{c \in C} p_{c}\|x\|_{c}$. By Lemma $2.1,\|\cdot\|$ is an equivalent norm on $X$. Since $0 \in C$ and since the sum of a strictly convex norm and any other equivalent norm is also strictly convex, $\|\cdot\|$ is strictly convex.

Remark. We have assumed that $X$ is a real Banach space. Similar results in the complex case can be obtained using

$$
\|y\|_{x}=\int_{0}^{2 \pi}\|\| y\left\|x+e^{-i \theta} y\right\| d \theta
$$

Our goal is to show that $\|\cdot\|$ satisfies the main theorem if $\|\cdot\|$ is strictly convex.

Lemma 2.2. Let $\|\cdot\|=\sum_{c \in C} p_{c}\|\cdot\|_{c}$ and let $\left(x_{n}\right) \subseteq X$ be $\|\cdot\|$-normalized.

a) If

$$
\lim _{m \rightarrow \infty} \lim _{n \rightarrow \infty}\left\|x_{m}+x_{n}\right\|=2 \lim _{m \rightarrow \infty}\left\|x_{m}\right\|,
$$

then there exists a subsequence $\left(x_{n}^{\prime}\right)$ of $\left(x_{n}\right)$ satisfying for all $y \in X$ and $\beta_{1}, \beta_{2} \geq 0$ that

$$
\lim _{m \rightarrow \infty} \lim _{n \rightarrow \infty}\left\|y+\beta_{1} x_{m}^{\prime}+\beta_{2} x_{n}^{\prime}\right\|=\lim _{m \rightarrow \infty}\left\|y+\left(\beta_{1}+\beta_{2}\right) x_{m}^{\prime}\right\| .
$$

b) If

$$
\lim _{m \rightarrow \infty} \lim _{n \rightarrow \infty}\left\|x_{m} \pm x_{n}\right\|=2 \lim _{m \rightarrow \infty}\left\|x_{m}\right\|,
$$

then there exists a subsequence $\left(x_{n}^{\prime}\right)$ of $\left(x_{n}\right)$ satisfying for all $y \in X$ and $\beta_{1}, \beta_{2} \in \mathbb{R}$ with $\left|\beta_{1}\right|+\left|\beta_{2}\right| \neq 0$ that

$$
\begin{aligned}
\lim _{m \rightarrow \infty} & \lim _{n \rightarrow \infty}\left\|y+\beta_{1} x_{m}^{\prime}+\beta_{2} x_{n}^{\prime}\right\| \\
& =\lim _{m \rightarrow \infty}\left(\left\|y \frac{\left|\beta_{1}\right|}{\left|\beta_{1}\right|+\left|\beta_{2}\right|}+\beta_{1} x_{m}^{\prime}\right\|+\left\|y \frac{\left|\beta_{2}\right|}{\left|\beta_{1}\right|+\left|\beta_{2}\right|}+\beta_{2} x_{m}^{\prime}\right\|\right) .
\end{aligned}
$$

Proof. a) We may choose $\left(x_{n}^{\prime}\right) \subseteq\left(x_{n}\right)$ so that for all $c \in C, y \in C$ and $\beta_{1}, \beta_{2} \in$ $[0, \infty) \cap \mathbb{Q}$ the limits

$$
\lim _{m \rightarrow \infty} \lim _{n \rightarrow \infty}\left\|y+\beta_{1} x_{m}^{\prime}+\beta_{2} x_{n}^{\prime}\right\|_{c}
$$

exist. Indeed this is easily done for fixed parameters and then one applies a diagonal argument.

Our hypothesis is that

$$
\lim _{m \rightarrow \infty} \lim _{n \rightarrow \infty} \sum_{c \in C} p_{c}\left\|x_{m}^{\prime}+x_{n}^{\prime}\right\|_{c}=2 \lim _{m} \sum_{c \in C} p_{c}\left\|x_{m}^{\prime}\right\|_{c} .
$$

Since $\lim _{m \rightarrow \infty}\left\|x_{m}^{\prime}\right\|_{c}$ exists, $\lim _{m \rightarrow \infty} \lim _{n \rightarrow \infty}\left\|x_{m}^{\prime}+x_{n}^{\prime}\right\|_{c}$ exists, $\left\|x_{m}^{\prime}+x_{n}^{\prime}\right\|_{c} \leq$ $\left\|x_{m}^{\prime}\right\|_{c}+\left\|x_{n}^{\prime}\right\|_{c}$ and $p_{c}\|\cdot\|_{c} \leq 2 p_{c}(1+\|c\|)\|\cdot\|$ for all $c \in C$, it follows that, since $\sum_{c \in C} p_{c}(1+\|c\|)<\infty$, for all $c \in C$,

$$
\lim _{m \rightarrow \infty} \lim _{n \rightarrow \infty}\left\|x_{m}^{\prime}+x_{n}^{\prime}\right\|_{c}=2 \lim _{m \rightarrow \infty}\left\|x_{m}^{\prime}\right\|_{c} .
$$

In particular taking $c=0$ we obtain that

$$
\lim _{m \rightarrow \infty} \lim _{n \rightarrow \infty}\left\|x_{m}^{\prime}+x_{n}^{\prime}\right\|=2 .
$$

It follows that since $\left\|x_{n}^{\prime}\right\|=1$,

$$
\lim _{m \rightarrow \infty} \lim _{n \rightarrow \infty}\left\|\beta_{1} x_{m}^{\prime}+\beta_{2} x_{n}^{\prime}\right\|=\beta_{1}+\beta_{2}
$$


for all $\beta_{1}, \beta_{2} \in[0, \infty)$. Similarly we have for all $c \in C$ and $\beta_{1}, \beta_{2} \in[0, \infty)$ that

$$
\lim _{m \rightarrow \infty} \lim _{n \rightarrow \infty}\left\|\beta_{1} x_{m}^{\prime}+\beta_{2} x_{n}^{\prime}\right\|_{c}=\left(\beta_{1}+\beta_{2}\right) \lim _{m \rightarrow \infty}\left\|x_{m}^{\prime}\right\|_{c} .
$$

Let $y \in C$ and $\beta_{1}, \beta_{2} \in[0, \infty) \cap \mathbb{Q}$ with $\beta_{1}+\beta_{2}>0$. Setting $c=\frac{y}{\beta_{1}+\beta_{2}}$ in (2) we obtain using (1) that

$$
\begin{aligned}
\lim _{m \rightarrow \infty} & \lim _{n \rightarrow \infty}\left(\left\|y+\beta_{1} x_{m}^{\prime}+\beta_{2} x_{n}^{\prime}\right\|+\left\|y-\beta_{1} x_{m}^{\prime}-\beta_{2} x_{n}^{\prime}\right\|\right) \\
& =\lim _{m \rightarrow \infty}\left(\beta_{1}+\beta_{2}\right)\left(\left\|\frac{y}{\beta_{1}+\beta_{2}}+x_{m}^{\prime}\right\|+\left\|\frac{y}{\beta_{1}+\beta_{2}}-x_{m}^{\prime}\right\|\right) \\
& =\lim _{m}\left(\left\|y+\left(\beta_{1}+\beta_{2}\right) x_{m}^{\prime}\right\|+\left\|y-\left(\beta_{1}+\beta_{2}\right) x_{m}^{\prime}\right\|\right) .
\end{aligned}
$$

The triangle inequality yields

$$
\begin{aligned}
\lim _{m \rightarrow \infty} & \lim _{n \rightarrow \infty}\left\|y \pm\left(\beta_{1} x_{m}^{\prime}+\beta_{2} x_{n}^{\prime}\right)\right\| \\
\leq & \lim _{m \rightarrow \infty}\left\|y \frac{\beta_{1}}{\beta_{1}+\beta_{2}} \pm \beta_{1} x_{m}^{\prime}\right\|+\lim _{n \rightarrow \infty}\left\|y \frac{\beta_{2}}{\beta_{1}+\beta_{2}} \pm \beta_{2} x_{n}^{\prime}\right\| \\
& =\lim _{m \rightarrow \infty} \beta_{1}\left\|\frac{y}{\beta_{1}+\beta_{2}} \pm x_{m}^{\prime}\right\|+\beta_{2}\left\|\frac{y}{\beta_{1}+\beta_{2}} \pm x_{m}^{\prime}\right\| \\
& =\lim _{m \rightarrow \infty}\left\|y \pm\left(\beta_{1}+\beta_{2}\right) x_{m}^{\prime}\right\| .
\end{aligned}
$$

Thus from (3) we have

$$
\lim _{m \rightarrow \infty} \lim _{n \rightarrow \infty}\left\|y+\beta_{1} x_{m}^{\prime}+\beta_{2} x_{n}^{\prime}\right\|=\lim _{m \rightarrow \infty}\left\|y+\left(\beta_{1}+\beta_{2}\right) x_{m}^{\prime}\right\| .
$$

This proves a) for $y \in C$ and $\beta_{1}, \beta_{2} \in \mathbb{Q} \cap[0, \infty)$, and hence by a density argument we obtain a) in general.

b) We may assume a) holds for $\left(x_{n}\right)$. The only remaining case we need consider is where $\beta_{1}>0$ and $\beta_{2}<0$. Actually we shall consider " $\beta_{1} x_{m}^{\prime}-\beta_{2} x_{n}^{\prime}$ " when $\beta_{1}, \beta_{2}>$ 0 . Arguing as above using $\lim _{m \rightarrow \infty} \lim _{n \rightarrow \infty}\left\|x_{m}-x_{n}\right\|=2 \lim _{m \rightarrow \infty}\left\|x_{m}\right\|$, we may assume that $\left(x_{n}\right)$ satisfies

$$
\lim _{m \rightarrow \infty} \lim _{n \rightarrow \infty}\left\|\beta_{1} x_{m}-\beta_{2} x_{n}\right\|_{c}=\left(\beta_{1}+\beta_{2}\right) \lim _{m \rightarrow \infty}\left\|x_{m}\right\|_{c}
$$

for all $c \in C$. Letting $y \in C$ and $c=\frac{y}{\beta_{1}+\beta_{2}}$ we obtain

$$
\begin{aligned}
\lim _{m \rightarrow \infty} & \lim _{n \rightarrow \infty}\left(\left\|y+\beta_{1} x_{m}-\beta_{2} x_{n}\right\|+\left\|y-\beta_{1} x_{m}+\beta_{2} x_{n}\right\|\right) \\
= & \left(\beta_{1}+\beta_{2}\right) \lim _{m \rightarrow \infty}\left(\left\|\frac{y}{\beta_{1}+\beta_{2}}+x_{m}\right\|+\left\|\frac{y}{\beta_{1}+\beta_{2}}-x_{m}\right\|\right) \\
= & \lim _{m \rightarrow \infty}\left(\left\|y+\left(\beta_{1}+\beta_{2}\right) x_{m}\right\|+\left\|y-\left(\beta_{1}+\beta_{2}\right) x_{m}\right\|\right) .
\end{aligned}
$$

Again by the triangle inequality we have

$$
\begin{aligned}
\lim _{m \rightarrow \infty} & \lim _{n \rightarrow \infty}\left\|y \pm\left(\beta_{1} x_{m}-\beta_{2} x_{n}\right)\right\| \\
\quad \leq & \lim _{m \rightarrow \infty}\left[\left\|y \frac{\beta_{1}}{\beta_{1}+\beta_{2}} \pm \beta_{1} x_{m}\right\|+\left\|y \frac{\beta_{2}}{\beta_{1}+\beta_{2}} \mp \beta_{2} x_{m}\right\| .\right.
\end{aligned}
$$


Since

$$
\begin{aligned}
\lim _{m \rightarrow \infty}\left[\left\|y \frac{\beta_{1}}{\beta_{1}+\beta_{2}}+\beta_{1} x_{m}\right\|+\left\|y \frac{\beta_{2}}{\beta_{1}+\beta_{2}}-\beta_{2} x_{m}\right\|\right. \\
\left.\quad+\left\|y \frac{\beta_{1}}{\beta_{1}+\beta_{2}}-\beta_{1} x_{m}\right\|+\left\|y \frac{\beta_{2}}{\beta_{1}+\beta_{2}}+\beta_{2} x_{m}\right\|\right] \\
=\lim _{m \rightarrow \infty}\left(\left\|y+\left(\beta_{1}+\beta_{2}\right) x_{m}\right\|+\left\|y-\left(\beta_{1}+\beta_{2}\right) x_{m}\right\|\right)
\end{aligned}
$$

it follows from (4) and (5) that we have

$$
\begin{aligned}
\lim _{m \rightarrow \infty} & \lim _{n \rightarrow \infty}\left\|y+\beta_{1} x_{m}-\beta_{2} x_{n}\right\| \\
\quad= & \lim _{m \rightarrow \infty}\left(\left\|y \frac{\beta_{1}}{\beta_{1}+\beta_{2}}+\beta_{1} x_{m}\right\|+\left\|y \frac{\beta_{2}}{\beta_{1}+\beta_{2}}-\beta_{2} x_{m}\right\|\right)
\end{aligned}
$$

which completes the proof of $b$ ).

Remark. In the language of types ([R1], $[\mathrm{M}])$ a) may be restated as

$$
\text { if } \quad \lim _{m \rightarrow \infty} \lim _{n \rightarrow \infty}\left\|x_{m}+x_{n}\right\|=2 \lim _{m \rightarrow \infty}\left\|x_{m}\right\| \text {, then }
$$

$\left(x_{m}\right)$ generates an $\ell_{1}^{+}$type on $(X,\|\cdot\|)$ (equivalently a double dual type).

The first part of our next lemma is not new (see e.g., [M], [R1]) but we include the proof for the sake of completeness. The second part is a slight twist of Maurey's result that a symmetric $\ell_{1}^{+}$-type yields $\ell_{1}$. In addition the second part of the next lemma establishes that b) of the main theorem holds for $\|\cdot\|$.

Lemma 2.3. Let $\left(x_{n}\right) \subseteq X$ be $\|\cdot\|$-normalized and let $\varepsilon_{i} \subseteq(0,1)$ decrease to 0 .

a) If

$$
\lim _{m \rightarrow \infty} \lim _{n \rightarrow \infty}\left\|x+\beta_{1} x_{m}+\beta_{2} x_{n}\right\|=\lim _{m \rightarrow \infty}\left\|x+\left(\beta_{1}+\beta_{2}\right) x_{n}\right\|
$$

for all $x \in X$ and $\beta_{1}, \beta_{2}>0$, then there exists a subsequence $\left(x_{n_{i}}\right)$ of $\left(x_{i}\right)$ satisfying for all $1 \leq i_{0} \leq k$ and $\left(\alpha_{i_{0}}, \alpha_{i_{0}+1}, \ldots, \alpha_{k}\right) \subseteq[0, \infty)$ that

$$
\left\|\sum_{i=i_{0}}^{k} \alpha_{i} x_{n_{i}}\right\| \geq\left(1-\varepsilon_{i_{0}}\right) \sum_{i=i_{0}}^{k} \alpha_{i} .
$$

In particular $\left(x_{n}\right)$ has no weakly null subsequence.

b) If

$$
\begin{aligned}
\lim _{m \rightarrow \infty} & \lim _{n \rightarrow \infty}\left\|x+\beta_{1} x_{m}+\beta_{2} x\right\| \\
\quad= & \lim _{m \rightarrow \infty}\left(\left\|y \frac{\left|\beta_{1}\right|}{\left|\beta_{1}\right|+\left|\beta_{2}\right|}+\beta_{1} x_{m}\right\|+\left\|y \frac{\left|\beta_{2}\right|}{\left|\beta_{1}\right|+\left|\beta_{2}\right|}+\beta_{2} x_{m}\right\|\right)
\end{aligned}
$$

for all $x \in X$ and $\beta_{1}, \beta_{2} \in \mathbb{R}$ with $\left|\beta_{1}\right|+\left|\beta_{2}\right| \neq 0$, then there is a subsequence $\left(x_{n_{i}}\right)$ so that for all $1 \leq i_{0} \leq k$ and $\left(\alpha_{i}\right)_{i_{0}}^{k} \subseteq \mathbb{R}$,

$$
\left\|\sum_{i=i_{0}}^{k} \alpha_{i} x_{n_{i}}\right\| \geq\left(1-\varepsilon_{i_{0}}\right) \sum_{i=i_{0}}^{k}\left|\alpha_{i}\right| .
$$

In particular $\left(x_{n_{i}}\right)$ is equivalent to the unit vector basis of $\ell_{1}$. 
Proof. a) Given $\delta_{i} \downarrow 0$ we can choose $\left(x_{n_{i}}\right) \subseteq\left(x_{i}\right)$ satisfying the following. For all $m<\ell$ and $y \in \operatorname{Ball}\left\langle x_{n_{i}}\right\rangle_{i=1}^{m-1}$, we have

$$
\left\|y+\beta_{1} x_{n_{m}}+\beta_{2} x_{n_{\ell}}\right\| \geq\left(1-\delta_{m}\right)\left\|y+\left(\beta_{1}+\beta_{2}\right) x_{n_{m}}\right\|
$$

if $\beta_{1}, \beta_{2} \in[0,1]$. Thus if $\left(\beta_{i}\right)_{i_{0}}^{k} \subseteq[0,1], \sum_{i_{0}}^{k} \beta_{i}=1$, then

$$
\begin{aligned}
\left\|\sum_{i=i_{0}}^{k} \beta_{i} x_{n_{i}}\right\| & =\left\|\sum_{i=i_{0}}^{k-2} \beta_{i} x_{n_{i}}+\beta_{k-1} x_{n_{k-1}}+\beta_{2} x_{n_{k}}\right\| \\
& \geq\left(1-\delta_{k-1}\right)\left\|\sum_{i=i_{0}}^{k-2} \beta_{i} x_{n_{i}}+\left(\beta_{k-1}+\beta_{k}\right) x_{n_{k-1}}\right\| \\
& \geq\left(1-\delta_{k-1}\right)\left(1-\delta_{k-2}\right)\left\|\sum_{1}^{k-3} \beta_{i} x_{n_{i}}+\left(\beta_{k-2}+\beta_{k-1}+\beta_{k}\right) x_{n_{k-2}}\right\| \\
& \geq \cdots \geq \prod_{i=i_{0}}^{k-1}\left(1-\delta_{i}\right)\left\|\sum_{i=i_{0}}^{k} \beta_{i} x_{n_{i_{0}}}\right\|=\prod_{i=i_{0}}^{k-1}\left(1-\delta_{i}\right) .
\end{aligned}
$$

a) follows if we choose the $\delta_{i}$ 's to satisfy $\prod_{i=i_{0}}^{\infty}\left(1-\delta_{i}\right) \geq 1-\varepsilon_{i_{0}}$ for all $i_{0}$. The "in particular" assertion is immediate from Mazur's theorem.

b) The argument here is similar but slightly more complicated than a) in as much as the condition in b) is not as nice as the one in a). Let $\delta_{i} \downarrow 0$ satisfy $\prod_{i=i_{0}}^{\infty}\left(1-\delta_{i}\right)>1-\varepsilon_{i_{0}}$ for all $i_{0}$ and using the assumption choose $\left(x_{n_{i}}\right) \subseteq\left(x_{i}\right)$ to satisfy for all $m<\ell$ and $y \in \operatorname{Ball}\left\langle x_{n_{i}}\right\rangle_{i=1}^{m-1}$,

$$
\begin{aligned}
& \left\|y+\beta_{1} x_{n_{m}}+\beta_{2} x_{n_{\ell}}\right\| \\
& \quad>\left(1-\delta_{m}\right)\left[\left\|\frac{\left|\beta_{1}\right|}{\left|\beta_{1}\right|+\left|\beta_{2}\right|} y+\beta_{1} x_{n_{m}}\right\|+\left\|\frac{\left|\beta_{2}\right|}{\left|\beta_{1}\right|+\left|\beta_{2}\right|} y+\beta_{2} x_{n_{m}}\right\|\right]
\end{aligned}
$$

if $\beta_{1}, \beta_{2} \in[-1,1]$ with $\left|\beta_{1}\right|+\left|\beta_{2}\right| \neq 0$.

We now show by induction on $k$ that

$$
\left\|\sum_{i=i_{0}}^{k} \beta_{i} x_{n_{i}}\right\| \geq \prod_{i=i_{0}}^{k-1}\left(1-\delta_{i}\right)
$$

if $i_{0} \leq k$ and $\sum_{i=i_{0}}^{k}\left|\beta_{i}\right|=1$. The claim is trivial for $k=1$ (taking $\left.\prod_{\phi}\left(1-\delta_{i}\right) \equiv 1\right)$. Assume validity of the claim for $k$ and let $\sum_{i=i_{0}}^{k+1}\left|\beta_{i}\right|=1$. For simplicity of the exposition assume $\beta_{i} \neq 0$ for $i_{0} \leq i \leq k+1$ (the general case follows by a density 
argument). Thus letting $y=\sum_{i=i_{0}}^{k-1} \beta_{i} x_{n_{i}}$ in (1),

$$
\begin{aligned}
\left\|\sum_{i=i_{0}}^{k+1} \beta_{i} x_{n_{i}}\right\| \geq\left(1-\delta_{k}\right) & {\left[\left\|\frac{\left|\beta_{k}\right|}{\left|\beta_{k}\right|+\left|\beta_{k+1}\right|} \sum_{i=i_{0}}^{k-1} \beta_{i} x_{n_{i}}+\beta_{k} x_{n_{k}}\right\|\right.} \\
& \left.+\left\|\frac{\left|\beta_{k+1}\right|}{\left|\beta_{k}\right|+\left|\beta_{k+1}\right|} \sum_{i=i_{0}}^{k-1} \beta_{i} x_{n_{i}}+\beta_{k+1} x_{n_{k}}\right\|\right] \\
\geq & \left(1-\delta_{k}\right) \prod_{i=i_{0}}^{k-1}\left(1-\delta_{i}\right)\left[\frac{\left|\beta_{k}\right|}{\left|\beta_{k}\right|+\left|\beta_{k+1}\right|} \sum_{i=i_{0}}^{k-1}\left|\beta_{i}\right|+\left|\beta_{k}\right|\right. \\
& \left.+\frac{\left|\beta_{k+1}\right|}{\left|\beta_{k}\right|+\left|\beta_{k+1}\right|} \sum_{i=i_{0}}^{k-1}\left|\beta_{i}\right|+\left|\beta_{k+1}\right|\right] \\
& =\prod_{i=i_{0}}^{k}\left(1-\delta_{i}\right) . \quad \square
\end{aligned}
$$

Lemma 2.4. Let $\|\cdot\|=\sum_{c \in C} p_{c}\|\cdot\|_{c}$, where $\|\cdot\|$ is an equivalent strictly convex norm on $X$. Let $\left(x_{n}\right) \subseteq X$ be a relatively weakly compact sequence. If

$$
\lim _{m \rightarrow \infty} \lim _{n \rightarrow \infty}\left\|x_{m}+x_{n}\right\|=2 \lim _{m \rightarrow \infty}\left\|x_{m}\right\|,
$$

then $\left(x_{n}\right)$ is norm convergent.

Proof. Since $\|\cdot\|$ is a strictly convex norm we need only show that $\left(x_{n}\right)$ has a convergent subsequence. Indeed if then $\left(x_{n}\right)$ were not convergent it would have two subsequences converging to $x \neq y$ respectively. But our hypothesis yields $\|x+y\|=2 \lim \left\|x_{m}\right\|=\|x\|+\|y\|$ which is impossible.

By passing to a subsequence of $\left(x_{n}\right)$ we may assume that $x_{n}=x+y_{n}$ where $\left(y_{n}\right)$ is weakly null and $\lim _{n \rightarrow \infty}\left\|y_{n}\right\|$ exists. If $\left(y_{n}\right)$ were not norm null, we may also assume $\left\|y_{n}\right\|=1$ for all $n$. From Lemma 2.2, passing to a further subsequence, we may assume that for all $y \in X$,

$$
\lim _{m \rightarrow \infty} \lim _{n \rightarrow \infty}\left\|y+x_{m}+x_{n}\right\|=\lim _{m \rightarrow \infty}\left\|y+2 x_{m}\right\| .
$$

For $z \in X$, letting $y=z-2 x$ we obtain

$$
\lim _{m \rightarrow \infty} \lim _{n \rightarrow \infty}\left\|z+y_{m}+y_{n}\right\|=\lim _{m \rightarrow \infty}\left\|z+2 y_{m}\right\| \text {. }
$$

Since in particular $\lim _{m \rightarrow \infty} \lim _{n \rightarrow \infty}\left\|y_{m}+y_{n}\right\|=2$ it follows from (1) and the definition of $\|\cdot\|$ that

$$
\lim _{m \rightarrow \infty} \lim _{n \rightarrow \infty}\left\|y_{m}+y_{n}\right\|=2 \lim _{m \rightarrow \infty}\left\|y_{m}\right\| .
$$

By Lemma 2.2 a) and Lemma 2.3 a) we conclude that $\left(y_{n}\right)$ is not weakly null which is a contradiction.

Summarizing our progress thus far we have shown that b) of the main theorem is satisfied for $\|\cdot\|=\sum_{c \in C} p_{c}\|\cdot\|_{c}$ and in addition a) holds if $\|\cdot\|$ is furthermore a strictly convex norm on $X$.

Lemma 2.5. Let $\|\cdot\|=\sum_{c \in C}\|\cdot\|_{c}$. If $\left(x_{n}\right) \subseteq X$ is weakly null and satisfies

$$
\lim _{m \rightarrow \infty} \lim _{n \rightarrow \infty}\left\|x_{m}+x_{n}\right\|=\lim _{m \rightarrow \infty}\left\|x_{m}\right\|>0,
$$

then $\left(x_{n}\right)$ admits a subsequence which is equivalent to the unit vector basis of $c_{0}$. 
Proof. Let $\left(x_{n}\right)$ satisfy the hypothesis of the lemma for $\|\cdot\|=\sum_{c \in C} p_{c}\|\cdot\|_{c}$. We may assume $\left(x_{n}\right)$ is basic, $\left\|x_{n}\right\|=1$ for all $n$, and that for all $y \in X$ and $\beta_{1}, \beta_{2} \in \mathbb{R}$ the following limits exist:

$$
\lim _{m \rightarrow \infty} \lim _{n \rightarrow \infty}\left\|y+\beta_{1} x_{m}+\beta_{2} x_{n}\right\| .
$$

Since $\left(x_{n}\right)$ is weakly null for all $y \in X$,

$$
\lim _{m \rightarrow \infty} \lim _{n \rightarrow \infty}\left\|x_{m}+x_{n}\right\|_{y} \geq \lim _{m \rightarrow \infty}\left\|x_{m}\right\|_{y} .
$$

As in the proof of Lemma 2.2 since

$$
\lim _{m \rightarrow \infty} \lim _{n \rightarrow \infty} \sum_{c \in C} p_{c}\left\|x_{m}+x_{n}\right\|_{c}=\lim _{m \rightarrow \infty} \sum_{c \in C} p_{c}\left\|x_{m}\right\|_{c}
$$

we obtain for all $y \in C$ and hence in $X$ that

$$
\lim _{m \rightarrow \infty} \lim _{n \rightarrow \infty}\left\|x_{m}+x_{n}\right\|_{y}=\lim _{m \rightarrow \infty}\left\|x_{m}\right\|_{y} .
$$

In particular, $\lim _{m \rightarrow \infty} \lim _{n \rightarrow \infty}\left\|x_{m}+x_{n}\right\|=1$. Thus by (1) for all $y \in X$,

$$
\begin{gathered}
\lim _{m \rightarrow \infty} \lim _{n \rightarrow \infty}\left(\left\|y+x_{m}+x_{n}\right\|+\left\|-y+x_{m}+x_{n}\right\|\right) \\
=\lim _{m \rightarrow \infty}\left(\left\|y+x_{m}\right\|+\left\|-y+x_{m}\right\|\right) .
\end{gathered}
$$

Since

$$
\lim _{m \rightarrow \infty} \lim _{n \rightarrow \infty}\left\| \pm y+x_{m}+x_{n}\right\| \geq \lim _{m \rightarrow \infty}\left\| \pm y+x_{m}\right\|
$$

we have from (2) that for all $y \in X$,

$$
\lim _{m \rightarrow \infty} \lim _{n \rightarrow \infty}\left\|y+x_{m}+x_{n}\right\|=\lim _{m \rightarrow \infty}\left\|y+x_{m}\right\| .
$$

Choose $\varepsilon_{i} \downarrow 0$ with $\prod_{1}^{\infty}\left(1+\varepsilon_{i}\right)<2$ and choose, using (3), a subsequence $\left(x_{n_{i}}\right)$ of $\left(x_{n}\right)$ so that for any integer $k \geq 0, k<i<j$, and $F \subseteq\{1, \ldots, k\}$ then

$$
\left\|\sum_{s \in F} x_{n_{s}}+x_{n_{i}}+x_{n_{j}}\right\| \leq\left(1+\varepsilon_{i}\right)\left\|\sum_{s \in F} x_{n_{s}}+x_{n_{i}}\right\| .
$$

It follows by iterating this inequality that for all finite $F \subseteq \mathbb{N},\left\|\sum_{s \in F} x_{n_{s}}\right\| \leq$ $\prod_{1}^{\infty}\left(1+\varepsilon_{i}\right)<2$. This implies that $\left(x_{n_{i}}\right)$ is equivalent to the unit vector basis of $c_{0}$.

Remark. The proof yields that for any $\varepsilon>0$ by judiciously choosing the $p_{c}$ 's and the original strictly convex norm one can choose the norm $\|\cdot\|$ satisfying the conclusion of the main theorem to satisfy for all $x \in X$,

$$
\|x\| \leq\|x\| \leq(1+\varepsilon)\|x\| .
$$

We give one final corollary of Lemma 2.5. Recall that the summing basis $\left(s_{n}\right)$ for $c_{0}$ is defined for all $n$ by $s_{n}=\sum_{i=1}^{n} e_{i}$.

Corollary 2.6. Let $\|\cdot\|=\sum_{c \in C} p_{c}\|\cdot\|_{c}$ and let $\left(x_{n}\right) \subseteq X$ satisfy

$$
\begin{gathered}
\lim _{n_{1} \rightarrow \infty} \lim _{n_{2} \rightarrow \infty} \lim _{n_{3} \rightarrow \infty} \lim _{n_{4} \rightarrow \infty}\left\|x_{n_{1}}-x_{n_{2}}+x_{n_{3}}-x_{n_{4}}\right\| \\
=\lim _{n_{1} \rightarrow \infty} \lim _{n_{2} \rightarrow \infty}\left\|x_{n_{1}}-x_{n_{2}}\right\|>0 .
\end{gathered}
$$

a) If $\left(x_{n}\right)$ is weak Cauchy but not weakly convergent, then some subsequence of $\left(x_{n}\right)$ is equivalent to the summing basis. 
b) If $\left(x_{n}\right)$ is weakly null, then some subsequence of $\left(x_{n}\right)$ is equivalent to the unit vector basis of $c_{0}$.

Proof. Lemma 2.5 yields the following. There exists $C<\infty$ so that for all subsequences of $\left(x_{n}\right)$ there exists a further subsequence $\left(y_{n}\right)$ so that for all finite $F \subseteq \mathbb{N}$,

$$
\left\|\sum_{n \in F}\left(y_{2 n}-y_{2 n-1}\right)\right\| \leq C \text {. }
$$

Let

$$
\mathcal{A}=\left\{\left(n_{i}\right) \in[\mathbb{N}]: \text { for all finite } F \subseteq \mathbb{N}, \quad\left\|\sum_{i \in F}\left(x_{n_{2 i}}-x_{n_{2 i-1}}\right)\right\| \leq C\right\} .
$$

Here $[\mathbb{N}]$ denotes the set of all subsequences of $\mathbb{N}$. $\mathcal{A}$ is a Ramsey set (see e.g., [O]) and thus by our remark above there exists $M \in[\mathbb{N}]$ so that $[M] \subseteq \mathcal{A}$. Thus by passing to a subsequence we may assume that if $n_{1}<\cdots<n_{2 k}$, then

$$
\left\|\sum_{1}^{k}\left(x_{n_{2 i}}-x_{n_{2 i-1}}\right)\right\| \leq C .
$$

a) By passing to a subsequence of $x_{n}$ we may assume that $\left(x_{n}\right)$ is basic and moreover $\left(x_{1}, x_{2}-x_{1}, x_{3}-x_{2}, \ldots\right)$ is seminormalized basic (see e.g., [Be, Theorem 8] or [R2]). Calling this sequence $\left(y_{n}\right)$ we have that $\left\|\sum_{n \in F} y_{n}\right\| \leq 2 C+\left\|x_{n_{1}}\right\|$ for all finite $F$, and so $\left(y_{n}\right)$ is equivalent to the unit vector basis of $c_{0}$. Hence $\left(x_{n}\right)$ is equivalent to the summing basis: $x_{n}=\sum_{i=1}^{n} y_{i}$.

b) By Elton's theorem (see [O]) we have that either a subsequence of $\left(x_{n}\right)$ is equivalent to the unit vector basis of $c_{0}$ or some subsequence $\left(y_{n}\right)$ of $\left(x_{n}\right)$ satisfies

$$
\lim _{k \rightarrow \infty}\left\|\sum_{i=1}^{k}(-1)^{i} y_{n_{i}}\right\|=\infty \text { for all } n_{1}<n_{2}<\cdots .
$$

From our above remarks we have that a subsequence is the unit vector basis of $c_{0}$.

Remark. 1. We do not know if $X$ can be given a norm $\|\cdot\|$ satisfying:

$$
\text { if } \quad \lim _{m \rightarrow \infty} \lim _{n \rightarrow \infty}\left\|x_{m} \pm x_{n}\right\|=\lim _{m \rightarrow \infty}\left\|x_{m}\right\|>0,
$$

then some subsequence of $\left(x_{n}\right)$ is equivalent to the unit vector basis of $c_{0}$.

We can show that this is the case for $\|\cdot\| \|$ provided in addition one has

$$
\lim _{m \rightarrow \infty} \lim _{n \rightarrow \infty}\left\|x_{m} \pm x_{n}\right\|=\lim _{m \rightarrow \infty} \| x_{m}
$$

However the hypothesis of Corollary 2.6 a) does require the assertion that $\left(x_{n}\right)$ not be weakly convergent. Indeed if $X$ contains $c_{0}$, then there exists a normalized sequence $\left(y_{n}\right) \subseteq X$ which is asymptotically 1-equivalent to the unit vector basis of $c_{0}$ and hence

$$
1=\lim _{n_{1} \rightarrow \infty} \lim _{n_{2} \rightarrow \infty}\left\|y_{n_{1}}-y_{n_{2}}\right\|=\lim _{n_{1} \rightarrow \infty} \lim _{n_{2} \rightarrow \infty} \lim _{n_{3} \rightarrow \infty} \lim _{n_{4} \rightarrow \infty}\left\|y_{n_{1}}-y_{n_{2}}+y_{n_{3}}-y_{n_{4}}\right\| .
$$

Thus $x_{n}=y+y_{n}$ satisfies the same condition for any $y \neq 0$ but $\left(x_{n}\right)$ admits no basic subsequence. 
2. As we have noted parts b) and c) of the main theorem hold for any equivalent norm $\|\cdot\|$ on $X$ where $\|\cdot\|=\sum_{c \in C} p_{c}\|\cdot\|_{c}$. From the proof of Lemma 2.4 it follows that whenever $\left(x_{n}\right) \subseteq X$ is relatively weakly compact and satisfies

$$
\lim _{m \rightarrow \infty} \lim _{n \rightarrow \infty}\left\|x_{m}+x_{n}\right\|=\lim _{m \rightarrow \infty}\left\|x_{m}\right\|,
$$

then $\left(x_{n}\right)$ is relatively norm compact.

\section{$\S 3$. Corollaries}

We now give some corollaries. Part a) of Corollary 3.1 yields a positive answer to Milman's problem mentioned above.

Corollary 3.1. Let $X$ be a separable Banach space. $X$ is reflexive (if and) only if there exists an equivalent norm $\|\cdot\|$ on $X$ satisfying the following for any bounded $\left(x_{n}\right) \subseteq X:$

a) If $\lim _{m \rightarrow \infty} \lim _{n \rightarrow \infty}\left\|x_{m}+x_{n}\right\|=2 \lim _{n}\left\|x_{n}\right\|$, then $\left(x_{n}\right)$ is norm convergent. Furthermore the norm $\|\cdot\|$ in a) satisfies

b) if $\left(x_{n}\right)$ is weakly null but not norm null, then

$$
\lim _{m \rightarrow \infty} \lim _{n \rightarrow \infty}\left\|x_{m}+x_{n}\right\|>\lim _{m \rightarrow \infty}\left\|x_{m}\right\|
$$

provided both limits exist.

Proof. The main theorem (a), c) ) yields such a norm if $X$ is reflexive. Conversely if a) holds let $x^{*} \in X^{*}$ with $\left\|x^{*}\right\|=1$. Choose $\left(x_{n}\right) \subseteq X,\left\|x_{n}\right\|=1$ with $\lim _{n \rightarrow \infty} x^{*}\left(x_{n}\right)=1$. It follows that $\lim _{m \rightarrow \infty} \lim _{n \rightarrow \infty}\left\|x_{m}+x_{n}\right\|=2$ and so by a), $\left(x_{n}\right)$ converges to some $x$ with $\|x\|=1$ and $x^{*}(x)=1$. Thus $x^{*}$ achieves its norm. By James' theorem [J1] $X$ must be reflexive.

Corollary 3.2. Let $X$ be a separable Banach space. Then there exists an equivalent norm $\|\cdot\|$ on $X$ such that if $Y$ is a subspace of $X$, then $Y$ is reflexive iff a) (and b)) of Corollary 3.1 hold for all bounded $\left(x_{n}\right) \subseteq Y$.

From b) and c) of the main theorem we obtain

Corollary 3.3. Let $X$ be a separable Banach space. The following are equivalent.

1) $X$ contains an isomorph of $\ell_{1}$ (respectively, $\left.c_{0}\right)$.

2) For all equivalent norms $\|\cdot\|$ on $X$ there exists a normalized sequence in $X$ having spreading model $\left(e_{n}\right)$ which is 1-equivalent to the unit vector basis of $\ell_{1}$ (respectively, $c_{0}$ ).

3) For all equivalent norms $\|\cdot\|$ on $X$ there exists a normalized (and respectively, weakly null) sequence in $X$ having spreading model $\left(e_{n}\right)$ satisfying $\left\|e_{1} \pm e_{2}\right\|=$ 2 (respectively $\left\|e_{1}+e_{2}\right\|=1$ ).

In addition to the main theorem the proof requires James' proof that $\ell_{1}$ and $c_{0}$ are not distortable ([J2] or [LT, p.97]). Indeed 1) $\Rightarrow 2$ ) or 3) is well known from James' result. Our discovery is the reverse implications.

Our work also yields the following corollaries.

Corollary 3.4. Let $X$ be a separable Banach space. The following are equivalent.

1) $X$ is not reflexive. 
2) For all equivalent norms $\|\cdot\|$ on $X$ there exists a $\|\cdot\|$-normalized basic sequence $\left(x_{i}\right)$ having spreading model $\left(\left(e_{i}\right),\|\cdot\|\right)$ satisfying for all $\left(a_{i}\right) \subseteq$ $[0, \infty)$

$$
\left\|\sum a_{i} e_{i}\right\|=\sum a_{i}
$$

3) For all equivalent norms $\|\cdot\|$ on $X$ there exists a $\|\cdot\|$-normalized basic sequence $\left(x_{i}\right)$ having spreading model $\left(\left(e_{i}\right),\|\cdot\| \|\right)$ satisfying

$$
\left\|e_{1}+e_{2}\right\|=2 \text {. }
$$

Corollary 3.5. Let $X$ be a separable Banach space. The following are equivalent.

a) $X$ is reflexive.

b) There exists an equivalent norm $\|\cdot\|$ on $X$ such that if $\left(\left(e_{i}\right),\|\cdot\|\right)$ is a spreading model of any $\|\cdot\|$-normalized basic sequence in $X$, then $1<$ $\left\|e_{1}+e_{2}\right\|<2$.

\section{REFERENCES}

[BL] B. Beauzamy and J.-T. Lapresté, Modèles étalés des espaces de Banach, Travaux en Cours, Herman, Paris, 1984. MR 86h:46024

[Be] S. Bellenot, Somewhat quasireflexive Banach spaces, Arkiv för matematik 22 (1984), 175-183. MR 86b:46014

[BP] C. Bessaga and A. Pełczyński, On bases and unconditional convergence of series in Banach spaces, Studia Math. 17 (1958), 151-164. MR 22:5872

[B] A. Brunel, Espaces associes à une suite bornée dans un espace de Banach, Séminaire Maurey-Schwartz, exposés 15, 16, 18, Ecole Polytechnique, 1973/4. MR 53:8866

[BS] A. Brunel and L. Sucheston, B-convex Banach spaces, Math. Systems Th. 7 (1974), 294299. MR 55:11004

[CS] P.G. Casazza and T.J. Shura, Tsirelson's Space, Lectures Notes in Math., vol. 1363, Springer-Verlag, Berlin and New York, 1989. MR 90b:46030

[D1] M.M. Day, Normed linear spaces, Springer-Verlag, New York, 1973. MR 49:9588

[D2] M.M. Day, Reflexive spaces not isomorphic to uniformly convex Banach spaces, Bull. Amer. Math. Soc. 47 (1941), 313-317. MR 2:221b

[DGZ] R. Deville, G. Godefroy and V. Zizler, Smoothness and Renormings in Banach Spaces, Pitman Mongraphs and Surveys in Pure and Applied Mathematics 64 (1993). MR 94d:46012

[FG] K. Fan and I. Glicksberg, Fully convex normed linear spaces, Proc. Nat. Acad. Sci. USA 41 (1955), 947-953. MR 17:386h

[FJ] T. Figiel and W.B. Johnson, A uniformly convex Banach space which contains no $\ell_{p}$, Comp. Math. 29 (1974), 179-190. MR 50:8011

[G1] W.T. Gowers, A new dichotomy for Banach spaces, Geom. Funct. Anal. 6 (1996), 10831093. CMP 97:04

[G2] W.T. Gowers, A space not containing $c_{0}, \ell_{1}$ or a reflexive subspace, Trans. Amer. Math. Soc. 344 (1994), 407-420. MR 94j:46024

[GM] W.T. Gowers and B. Maurey, The unconditional basic sequence problem, J. Amer. Math. Soc. 6 (1993), 851-874. MR 94k:46021

[HM] R. Haydon and B. Maurey, On Banach spaces with strongly separable types, J. London Math. Soc. (2) 33 (1986), 484-498. MR 87g:46026

[J1] R.C. James, Reflexivity and the sup of linear functionals, Israel J. Math. 13 (1972), 289-300. MR 49:3506

[J2] R.C. James, Uniformly nonsquare Banach spaces, Ann. Math. 80 (1964), 542-550. MR 30:4139

[J3] R.C. James, Bases and reflexivity of Banach spaces, Annals of Math. 52 (1950), 518-527. MR 12:616b

[KM] J.L. Krivine and B. Maurey, Espaces de Banach stables, Israel J. Math. 39 (1981), 273295. MR 83a:46030 
[LT] J. Lindenstrauss and L. Tzafriri, Classical Banach Spaces I, Springer-Verlag, New York, 1977. MR 58:17766

[M] B. Maurey, Types and $\ell_{1}$-subspaces, Longhorn Notes, Texas Functional Analysis Seminar 1982-83, The University of Texas at Austin, 123-137.

[MMT] B. Maurey, V.D. Milman and N. Tomczak-Jaegermann, Asymptotic infinite-dimensional theory of Banach spaces, Operator Theory: Advances and Applications 77 (1995), 149175. MR 97g:46015

[Mil] D.P. Milman, On some criteria for the regularity of spaces of the type (B), Dokl. Akad. Nauk SSSR 20 (1938), 243-246, (in Russian).

[Mi] V.D. Milman, Geometric theory of Banach spaces II, geometry of the unit sphere, Russian Math. Survey 26 (1971), 79-163, (trans. from Russian). MR 54:8240

[MT] V.D. Milman and N. Tomczak-Jaegermann, Asymptotic $\ell_{p}$ spaces and bounded distortion, (Bor-Luh Lin and W.B. Johnson, eds.), Contemporary Math. 144 Amer. Math. Soc. (1993), 173-195. MR 94m:46014

[O] E. Odell, Applications of Ramsey theorems to Banach space theory, Notes in Banach spaces (H.E. Lacey, ed.), U.T. Press, Austin, pp. 379-404. MR 83g:46018

[OS] E. Odell and Th. Schlumprecht, A problem on spreading models, to appear in J. Funct. Anal.

[P] B.J. Pettis, A proof that every uniformly convex space is reflexive, Duke Math. J. 5 (1939), 249-253.

[R1] H. Rosenthal, Double dual types and the Maurey characterization of Banach spaces containing $\ell^{1}$, Longhorn Notes, Texas Functional Analysis Seminar 1983-84, The University of Texas at Austin, 1-37. CMP 18:10

[R2] H. Rosenthal, A characterization of Banach spaces containing $c_{0}$, J. Amer. Math. Soc. 7 (1994), 707-747. MR 94i:46032

[R3] H. Rosenthal, A characterization of Banach spaces containing $\ell^{1}$, Proc. Nat. Acad. Sci. USA 71 (1974), 2411-2413. MR 50:10773

[T] B.S. Tsirelson, Not every Banach space contains $\ell_{p}$ or $c_{0}$, Funct. Anal. Appl. 8 (1974), 138-141.

Department of Mathematics, The University of Texas at Austin, Austin, Texas 787121082

E-mail address: odell@math.utexas.edu

Department of Mathematics, Texas A\&M University, College Station, Texas 778433368

E-mail address: schlump@math.tamu.edu 